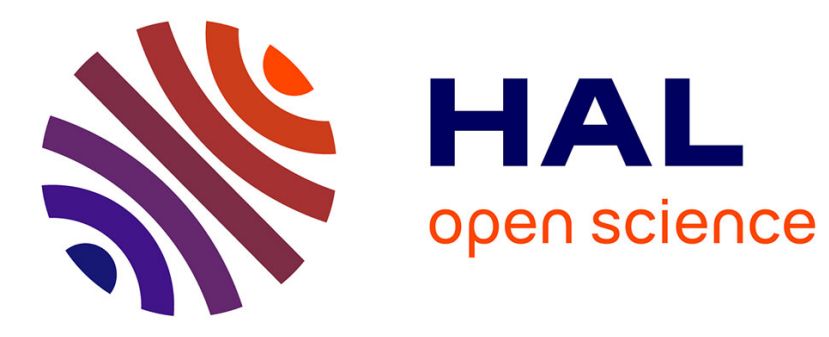

\title{
Multi-channel Distributed MAC protocol for WSN-based wildlife monitoring
}

\author{
Viktor Toldov, Laurent Clavier, Nathalie Mitton
}

\section{To cite this version:}

Viktor Toldov, Laurent Clavier, Nathalie Mitton. Multi-channel Distributed MAC protocol for WSNbased wildlife monitoring. WiMob 2018 - 14th International Conference on Wireless and Mobile Computing, Networking and Communications, Oct 2018, Limassol, Cyprus. hal-01866809

\section{HAL Id: hal-01866809 \\ https://hal.inria.fr/hal-01866809}

Submitted on 3 Sep 2018

HAL is a multi-disciplinary open access archive for the deposit and dissemination of scientific research documents, whether they are published or not. The documents may come from teaching and research institutions in France or abroad, or from public or private research centers.
L'archive ouverte pluridisciplinaire HAL, est destinée au dépôt et à la diffusion de documents scientifiques de niveau recherche, publiés ou non, émanant des établissements d'enseignement et de recherche français ou étrangers, des laboratoires publics ou privés. 


\title{
Multi-channel Distributed MAC protocol for WSN-based wildlife monitoring
}

\author{
Viktor Toldov ${ }^{1,2}$, Laurent Clavier $^{2,3}$, Nathalie Mitton ${ }^{1}$ \\ ${ }^{1}$ Inria firstname.lastname@inria.fr, ${ }^{2}$ IRCICA USR CNRS 3380, Université Lille 1, IEMN, \\ ${ }^{3}$ Institut Mines-Télécom, Télécom-Lille, firstname.lastname@telecom-lille.fr
}

\begin{abstract}
Several wild animal species are endangered by poaching. As a solution, deploying wireless sensors on animals able to send regular messages and also alert messages has been envisaged recently by several authorities and foundations. In that context, this paper proposes WildMAC, a multichannel, multihop wireless communication protocol for these specific wireless sensor networks that have to collect data from unknown large areas with different QoS requirements. WildMAC is a TDMA based MAC protocol that leverages long range communication properties to propose an efficient data collection mean. Its performance evaluation shows it meets QoS requirements.
\end{abstract}

\section{INTRODUCTION}

The current threat to the natural environment and biodiversity is serious and increasing rapidly. One particular area of concern is the drastic reduction in numbers of specific African species, such as elephant, rhinoceros, pangolin and lion. These are by no means all and not restricted to Africa either. However, in the case of rhinoceros, the situation is particularly severe. The Javan and Sumatran species are critically endangered with very low numbers remaining. In Vietnam a subspecies of the Javan rhinoceros has been hunted to extinction in $2011^{1}$. From an African perspective, things are still better for some species, but deteriorating fast. The Northern White- and Western Black rhinos have both been declared extinct in the wild. Similarly, there is severe poaching pressure on all other African species and subspecies. In Southern Africa, the careful protection and conservation of rhino during the last 50 years have resulted in a healthy increase in Southern White and Black rhino totals. However, this is now turning around with illegal trade in rhino horn resulting in very high poaching losses ${ }^{2}$. Most of these have been poached in big Parks like Kruger National Park and in KwaZulu-Natals areas, but also significantly on private land and game areas. The annual natural increase in numbers is now very close to, if not already being exceeded, by the number poaching. Current accelerating loss statistics leave no doubt that the end result will be catastrophic for species survival. Motivated to attempt making a contribution, a research and development group has been formed between researchers in France and in South Africa ${ }^{3}$. The idea is to deploy a wireless sensor network over rhinos. The sensors carried by rhinoceros embed

\footnotetext{
${ }^{1}$ http://www.worldwildlife.org/species/rhino

${ }^{2}$ https://www.savetherhino.org/rhino-info/poaching-stats/

${ }^{3}$ https://iww.inria.fr/prednet/en/
}

different sensors including GPS, accelerometer, etc able to detect an abnormal behavior of the animal. The project features different aspects ranging from the design of this sensor, the identification of the animal behavior based on sensor data to the data collection. This paper focuses on the data collection and wireless communication.

In this paper, we propose WildMAC. WildMAC is a multichannel distributed slotted Aloha-based protocol with slot reservation and multi-hop capabilities. It is a synchronized TDMA based solution providing the long-term time-slot reservation for the nodes in the proximity of the sink nodes and on-demand one-time slot reservation for the nodes which are not the neighbors of the sink. The TDMA approach enables to decrease the competition between the nodes in the proximity of the base stations (BTS) or sink nodes. We have evaluated the performance of WildMAC and the results show that for a proper system sizing, WildMAC delivers regular and alert messages within the QoS requirements set by the wildlife monitoring.

The remaining of the paper is organized as follows. Section II states the problem and motivates our assumptions. Section III presents literature work on the used of wireless sensors for wildlife monitoring and usual MAC layers. It brows the strengths and lengths of these approaches and explains how they inspired WildMAC. Section IV details the WildMAC protocol which is then evaluated in Section V. Finally, Section VI concludes this work.

\section{PROBLEM STATEMENT AND ASSUMPTION}

As mentioned, we focus on an adaptive communication protocol able to collect data from wild animals in a large area such as the Kruger park. An area as large as the Kruger park can be covered by a set of base stations equipped with long range communication technologies such as LoRa [1], [2] or SigFox [3] that can reach several dozens of kilometers range. However, in a wild and hostile area, base stations can not be deployed everywhere for energy and security issues. They must be settled in camps. Our previous work [4] has shown that even the long range offered by long range communications technologies, this is not possible to entirely cover the whole area. There is thus a need for multi-hop routing protocol.

Based on the requirements expressed by rangers and zoologists, several vitals of the animals should be regularly collected for monitoring purpose. Using a long range communication allows the coverage of a large area but also of a significant 
number of animals. Our previous work [5] has shown that a single channel solution is not enough to absorb the whole traffic. There is thus a need for multi-channel protocol.

The communication system must support two kinds a traffic: periodic monitoring traffic and data alerts. Both traffics have different delay requirements. A regular message is expected once a hour while the alert message should arrive within $60 \mathrm{~s}$.

Finally, the system must be energy efficient. Limited size and weight of devices impose limitation of capacity of the battery, and, thus, available energy. Moreover, these batteries are not expected to be changed.

To the best of our knowledge, none of existing MAC layer protocols meet all these requirements at once. Thus, the development of a new MAC layer protocol is needed.

Capture effect. The design of WildMAC assumes the capture effect to occur at the physical layer. The capture effect is a phenomenon common to most of wireless technologies and assume that in case of reception of several simultaneous signals on a same channel, the one with the strongest signal-to-noise ratio will be successfully demodulated. Such a phenomenon has been confirmed with the use of LoRa technology and some others [6], [7]. When 2 LoRa packets are sent to the same receiver with the same communication parameters and partially overlap in time, only the first packet will be received if the transmission of the second packet starts after the end of the preamble of the first one. The second packet in this case will be ignored and the first packet can still be correctly decoded. This phenomenon happens even if the Received Signal Strength (RSS) of the second packet at the receiver side is higher than the RSS of the first packet until a specific limit which depends on the used SF [8].

\section{RELATED WORK}

\section{A. Rhino tracking with wireless technology}

Several initiatives have been launched to track and protect rhinoceros by using wireless technology [9]-[11]. All of them relies on long range (LPWAN) technologies as ours for the same reasons and constraints as detailed in Section II. However, so far, they all target private reserves or national parks that are smaller than the Kruger Park. They are thus able either to reach every rhino in a single hop communications or to deploy BTS wherever they need to achieve this single-hop communication.

\section{B. Wildlife tracking solutions based on wireless technology}

Other wildlife tracking projects are described in literature. Even though the final aim of these projects is similar to the rhinoceros preservation project, there are several differences in the concept and do not always face the same constraints. For example, ZebraNet project [12] aims to track wild animals in central Kenya. Although the project mainly focuses on zebras, the developed network could be applied for different kinds of animals. The authors emphasis the fully mobile nature of the sensor network developed within the project. Not only end nodes (devices, carrying by zebras), but also base stations (the data sinks) are considered as mobile. The challenge of this solution is the unknown time of the BTS availability. It is supposed that the base station can arrive close to the animals between noon and midnight. The nodes, thus have to search for a BTS during a potential long time which is energy consuming. Moreover, that introduces huge delays in data collection (at least 12 hours). These factors make this solution unsuitable to meet the requirements of our case (see Section II). This delaytolerant feature also makes ZebraNet solution unsuitable for alarm mode operation.

In [13], [14], an ultra low power WSN for tracking bats in the wild is proposed. Small size of the bats strictly limits acceptable maximum weight of the carried nodes to 2 grams including battery. Small battery size limits the amount of available energy in the node. To meet the energy limitations and lower the energy consumption of the nodes, a low power wakeup receiver is integrated in the carried by the bats devices. The collected data is sent to the ground base station when a bat carrying the node flies close enough to this latter. When it happens, the wake-up signal (which is sent periodically by the base station) is received by the low power receiver in order to activate the main transmitter and send the data. Small communication range with ground stations (about $50 \mathrm{~m}$ ) leads to spontaneous communications in the system. Relatively high mobility of the bats along with small monitoring area (comparatively to the Kruger park) make the communication opportunity happens often. However, impossibility to cover all the habitat area in the Kruger park and relatively low mobility of rhinoceros would cause high delays for message transmissions, which is unacceptable for the alarm messages.

A similar application for flying foxes (fruit bats) is described in [15]. The authors propose a 3-tier system containing mobile nodes installed on the animals, gateways and cloud service. In this case, the multihop communications are not provided which makes impossible a rapid delivery of alarm messages from uncovered areas. However, authors propose to adapt the behavior of the nodes depending on the current state of charge which can evolve due to both, energy consumption and energy harvesting. This option can be useful in our project and potentially reused.

In [16], an inverse-GPS tracking system for birds and small mammals is proposed. The system allows localizing effectively the animals within large areas. However, the project is not focused on data collection and transmissions. Redundant coverage of the area is required in the proposed system, which is not always possible in the Kruger park.

Another project proposes a solution to monitor migrating whooping cranes [17] with less sensors. However, it relies on GSM cellular technology with another short range 802.15.4 radio to send the data, which are not suitable in our case.

Finally, some wildlife animal tracking solutions are based on the satellite technologies (e.g., Argos, Iridium, Globalstar) [18], [19]. Even though these solutions ensure a good coverage of the target area, they have important drawbacks. First, the hardware installed on the animals is very expensive, which makes impossible to equip all the animals in the target area. Second, the energy consumption of these devices is high, which leads to the short lifetime of the nodes. 


\section{MAC protocols}

The LoRa technology already comes with a MAC layer called LoRaWAN [20]. LoRaWAN uses a simple sender initiated ALOHA-based MAC protocol. The advantage of this solution is that it does not require development of any Radio Duty Cycle (RDC) strategy: the nodes can start their transmissions at any moment. Since, the sink node (the Base station) is always on and listening to all the available channels, the message could be received immediately, without any connection establishment process. However, this approach requires the full coverage of the target zone, which, is not possible in our case.

Different MAC protocols are proposed in literature. Well known MAC protocols used in WSN with IEEE 802.15.4 compatible radios (e.g. X-MAC [21], ContikiMAC [22]) do not directly fit our requirements due to their single-channel nature. The ORPL [23] proposes a ContikiMAC based solution to decrease the overall energy consumption of the WSN, based on anycast transmissions. The messages are accepted and ACKed only by nodes, situated closer to the sink node. However, to define, whether the node is closer to the sink or not, the RPL routing protocol [24] is used to define the rank (the number of hops that the message should pass to arrive to the sink). RPL is designed for static topologies, thus will not perform well in our case. Moreover, it is resource consuming in terms of memory. In [25], authors propose a Slotted Seeded Channel Hopping ( $\mathrm{SSCH}$ ) protocol that uses multiple channels to increase the capacity of the network for both single-hop and multi-hop scenarios. The SSCH protocol is designed to be used with only one radio module per node, while a number of multi-channel MAC protocols need to use 2 radios [26]. However, this protocol is originally designed for IEEE 802.11 wireless cards which are supposed to stay in active mode all the time. So, the SSCH protocol could not be used even with low-power radios because of a lack of the sleep mode (energy constraint). Moreover, the SSCH protocol assumes high throughput wireless links of 802.11 cards which enables to use up to 35 maximum length packets within a one timeslot. These communication rates are not available for long range low power transceivers. The Timeslotted Channel Hopping (TSCH) is a mode of IEEE 802.15.4e [27] standard describing time-slotted communications between WSN nodes with channel hopping. This standard focuses on communication mechanisms, but do not describe strategies to build and maintain communication schedules. Communication schedules maintaining for mobile networks is challenging. The schedule can vary in different locations. Indeed, the same timeslots can be reused in different geographical zones. We believe that time-slotted structure, as proposed in $\mathrm{TSCH}$, is useful for our project application, since it helps to decrease energy loss due to the connection establishment process and helps to manage contention based access to the medium. However, the channel hopping approach as a solution against interference is questionable because of the long on-air time for long range transmission technologies, which leads to long timeslots along with long application layer packet generation period. The AMAC protocol [28] proposes to adapt the WSN node duty cycle according to the current battery State of Charge. It can be useful in our case. Indeed, nodes with higher amount of available energy can wake-up more often in order to forward messages from neighbors, which will decrease the end-to-end delay. At the same time, the nodes with less energy available wake up less often in order to save energy.

To sum up, many MAC layer protocols have been proposed in literature but to the best of our knowledge, none meets all requirements of our wildlife application. Therefore, we propose WildMAC that leverage strengths of existing protocols to provide an efficient long-range based mutichannel multihop wireless MAC protocol.

\section{WILDMAC}

To meet all the requirements previously defined in Section II, WildMAC is a multi-channel distributed slotted Alohabased protocol with slot reservation and multi-hop capabilities that provide two communication modes : (i) alarm mode and (ii) plain monitoring mode. It is a synchronized TDMA-based solution providing the long-term time-slot reservation for the nodes in the proximity of the sink nodes and on-demand onetime slot reservation for the nodes which are not the neighbors of the sink. Synchronization is ensured by the GPS chip embedded on all sensors and providing a common clock. The TDMA approach enables to decrease the competition between the nodes in the proximity of the base stations (BTS) or sink nodes. It is important, since these nodes have to forward the messages from all other nodes which are out of range of the BTS. Thus, the synchronization is required to maintain the time-slotted structure. WildMAC relies on a gradient routing rooted at the based station.

\section{A. Overall idea}

Each node $u$ is aware of its distance in number of hops to the base station that we refer as its rank (BTS has rank 0). Nodes will then use a channel depending on their rank, limiting interferences. We divide the time into super frames composed of $n_{T S}$ Time Slots. The duration $d$ of a Super Frame is equal to the application data generation period, in order to ensure that a message emitted from somewhere in the network can reach the BTS within this given period. Each timeslot is divided in 3 parts: Control part, First data exchange part (1st TX/RX) and Second data exchange part (2nd TX/RX) that we detail below.

Control part is dedicated to alarm transmission and rank discovery. It is built in such a way that alarm transmission is made priority in each time slot, ensuring that it reached the BTS as fast as possible. Other parts (Tx/Rx parts) are used for plain monitoring, e.g slot reservation and data transmission.

\section{B. Control part}

Rank discovery. The rank discovery can be performed within the control part of each time slot. Indeed, each node can broadcast its own rank if known during the control part he timeslot after a fixed offset. This fixed offset depends of the rank to advertise, it is proportional to the Rank value, i.e. the beacons from lower ranked nodes are sent before higher ranked 
nodes. In the case of use of a generic radio, the offset step between differently ranked nodes could be set to the duration of the beacon message. In this case, the beacon messages will be sent consecutively starting from BTS nodes $(\mathrm{Rank}=0)$ to the furthest nodes.

Supposing a new node situated 3 hops away from the BTS, wishing to join the network. To determine its Rank, node C listens for beacon messages during the control part. The node is able, thus, to receive the beacon messages from Rank 2 nodes, other Rank 3 nodes and Rank 4 nodes, since these nodes can be in the communication range of $\mathrm{C}$. The Rank is defined then by the earliest received beacon message. In order to save energy, the node does not even need to decode the message and thus even in case of collision, it can estimate its rank.

\section{Alarm transmission.}

As can be noticed from Fig. 1, the offset of the Rank 0 (BTS) beacon message is greater than zero and equals to the duration of the preamble, as the offset in other cases. This enables the transmission of the urgent alarm messages. Indeed, alarms can be sent at offset 0 so earlier than all beacon messages. This gives these alarm messages a higher priority at the physical layer due to the capture effect described above. The alarm messages can be confirmed by an Acknowledgement message in order to ensure reliable transmissions.

1) First and Second TX/RX parts: These sections are dedicated to the sending and forwarding of regular monitoring information, based on a slot reservation mechanism.

Each node uses one of the TX/RX parts to transmit data and another to receive, based on its rank. In the example presented in Figure 1 the nodes with the even Rank values $(0,2,4)$ receive data packets from the upper ranked nodes during the first TX/RX part (reception function) and transmit packets to lower ranked nodes during second TX/RX part (transmission function). For odd ranked nodes $(1,3,5)$, the functions are opposite: transmission function for the first TX/RX part and receive function for the second one.

Each node accesses the medium at a given timeslot in each superframe. This given timeslot is reserved by a node, through a permanent reservation for Rank 1 nodes (once a node has a confirmed timeslot number, it keeps it), or upon a on-demand reservation for higher rank nodes. These reservation mechanisms are defined below. The data messages sent during first or second TX/RX parts are acknowledged by ACK messages. We can notice that in Figure 1, the First TX/RX part is slightly longer than the second one. The second TX/RX part is shorter due to the ACK message which was moved to the Control part of the next timeslot, as mentioned before. It helps to optimize the timeslot duration.

\section{Multichannel operation structure}

The multichannel operation of the protocol is proposed in order to improve communication performance. However, to enable multichannel operation we assume that BTS is able to receive messages from multiple channels simultaneously. Using several channels allows for a larger supported number of hops but also for less inter-rank nodes interferences. Channels are managed as illustrated on Fig. 1.

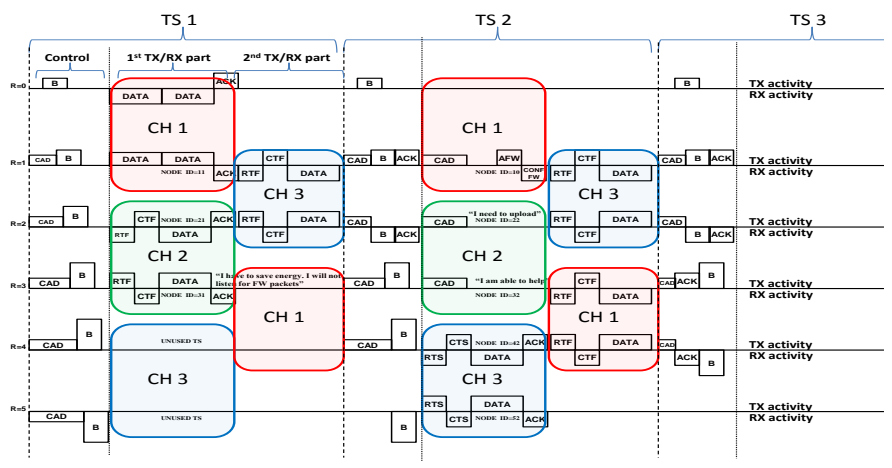

Fig. 1. Inter-rank frequency division structure

On Fig. 1, different channels are represented as different colors. The presented structure enables separation between parallel inter-rank transmissions in a pairwise way. Thus, for the mentioned example, Rank 2 node receives the packet from Rank 3 node via the channel 2 during the first TX/RX part. The transmission of a neighboring Rank 1 node to the BTS in this case will not have any impact on Rank 2 node, since Rank 1 to 0 transmission is isolated in the other channel - channel 1. In the same way, the transmission between Rank 5 and 4 is isolated to the channel 3. Similar logic is applied to the 2nd TX/RX part of the timeslot.

\section{Timeslot reservation and channel access}

If the problem of inter-rank interference can be solved by implementing the multichannel strategy, the intra-rank interference issue should be managed. To do so, we propose a permanent timeslot reservation mechanism for Rank 1 nodes and competition based channel access strategy for the nodes with Rank greater than 1. We later call this challenge based channel access as One-time timeslot reservation. The competition is based on Request To Forward/Clear To Forward (RTF/CTF) messages exchange. Let's suppose that two Rank $n$ nodes willing to send data message up, to the neighboring Rank $n-1$ node (As nodes 21 and 22 on Fig. 2). Since the network is synchronized, all Rank $n$ nodes will send the RFT messages simultaneously (concurrently). We rely here on the capture effect, as described in [6]. At least one Rank $n-1$ node will successfully receive the message received with the strongest RSS and reply with a CTF message containing the address of the rank- $n$ node whose RTF message was successfully decoded (winner of the competition). Since all Rank $n$ nodes in range, participating to the competition, receive the CTF message, each participant is aware whether it can send the data packet or it has to retry the competition during the next timeslot. A node will answer a CTF by a RTF if and only if it has enough energy to help in data transmission of other nodes.

All nodes with a Rank greater than 1 have to participate in the described competition each time they need to transmit a packet. It means that RTF/CTF message exchange must always be carried out before data transmission. To avoid the bottleneck problem at the Rank 1 nodes due to a larger number 


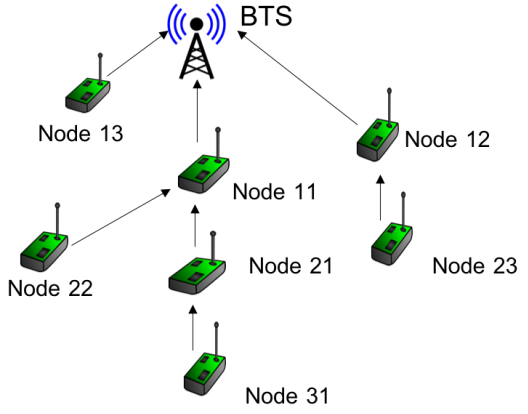

Fig. 2. Example of a topology built for WildMac

of messages to send (A Rank 1 node must send its own data but also forward data from higher rank nodes), we propose a long term timeslot reservation technique. After timeslot reservation, Rank 1 nodes do not need to exchange the RTF/CTF messages each time they need to transmit data to the BTS. The onair-time which is not used now for RTF/CTF messages can be reused for additional data packet transmission, which will help to free the packet buffer quickly and avoid the bottleneck problem.

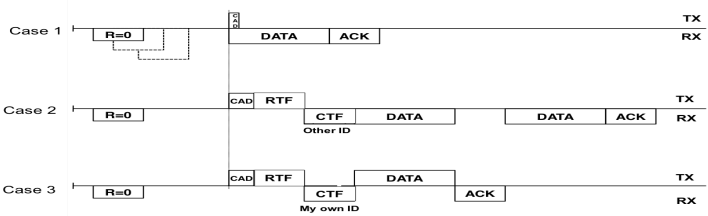

Fig. 3. One-time slot reservation for Rank 1 nodes

The reservation process is depicted in Fig. 3 from a rank1 node's perspective. When it wishes to access the medium for the first time, the following cases can occur: either) the channel is already used by another rank-1 node: the node waits for the next time slot; or) no activity is detected: all nodes willing to reserve the timeslot enter the competition similar to the one described before. All the reserving nodes, send a RTF message concurrently to a rank- $n-1$ node. As in the competition for higher ranks nodes, the winner of the competition will be defined on the physical layer thanks to the capture effect. The winner will be announced as before by the Clear To Forward (CTF) message. If a competing node receives a CTF that contains its own ID, it can send its data immediately after. If the RTF contains another ID, it waits for the next time slot.

\section{E. Additional transmissions}

1) Upon request: In case of high traffic, Rank 1 nodes can also request one-time use timeslot based on the competition similar to one for higher ranks. To avoid the interference problems, a specific channel and time offset should be used for this purpose, as depicted in the Fig. 4. We propose to use the same channel for one-time timeslot reservation transmission of Rank 1 nodes and communication between Rank 2 and 3 nodes. However, the proposed offset ensures that even though the Rank 1 node (node ID 10 in the figure) and Rank 2 node (node ID 21 in the figure) are within the communication range and sharing the same channel, the transmissions are always carried out in the opposite directions and so cannot interfere at the receiver. As shown in the picture with a dotted line, when node 10 sends an RTF message to the BTS and node 21 sends a CTF message back to the Rank 3 node (ID 31), neither BTS, nor node 31 are impacted by opposite transmissions, since Rank 3 and Rank 0 nodes are not in communication range. Similar situation is valid for the reception: node 10 cannot receive messages from node 31 , as well as BTS cannot receive messages from the node 21 since these nodes are far from each other. The communication, thus, are isolated.

The one-time slot reservation for Rank 1 nodes allows the node to send up to 2 data packets with ACK messages. In this case both, 1st and 2nd TX/RX parts are used. This option also helps to avoid a bottleneck problem at the Rank 1 nodes, as it gives the opportunity to offload the packets from the buffer in case of overload.

2) Upon invitation: In case of high traffic, based on the different energy level of nodes, WildMAC proposes an additional mechanism to allow nodes that have no data to send to alleviate overloaded neighboring nodes. To do so, after checking that the channel is free and the timeslot can thus be used for offloading data packets, a free rank-1 node that has enough energy left announces its availability to forward new packets by sending an AFW (available to forward) message to the BTS during the first TX/RX part of the slot. The BTS confirms its candidature by a forward confirmation message (CONF FW) to define one Rank 1 node per timeslot in case of multiple nodes available to forward messages. The winner node can thus answer RTF from rank-2 nodes if any during the second TX/RX part of the time slot, as illustrated on Figure 4.

\section{F. Communication example}

In Fig. 4, an example of communication is provided in order to help the understanding of the WildMAC protocol with respect to topology shown on Fig.2.

For the sake of simplicity, only one time division structure is shown in this example. We suppose also that all the nodes in the figure have already determined their rank and Rank 1 nodes have reserved their timeslots. In the figure, Node 31 (Rank 3) is willing to offload the data from its buffer during the 1 st TX/RX part of the timeslot 1 . It thus requests an access to the timeslot via the competition mechanism described before. Node 21 (Rank 2) confirms the success of Node 31 by sending back a CTS message. Then the data are sent to Node 21 and confirmed by an ACK message. As defined, this communication was done on Channel 2. During the 2nd TX/RX part of the timeslot, Node 21 switches to Channel 3 in order to forward the received packet, or a packet with its own data to Rank 1 node. Node 21 participates in the competition as done by Node 31, and Node 11 confirms the success of Node 21 and receives its data packet. This time the ACK message 


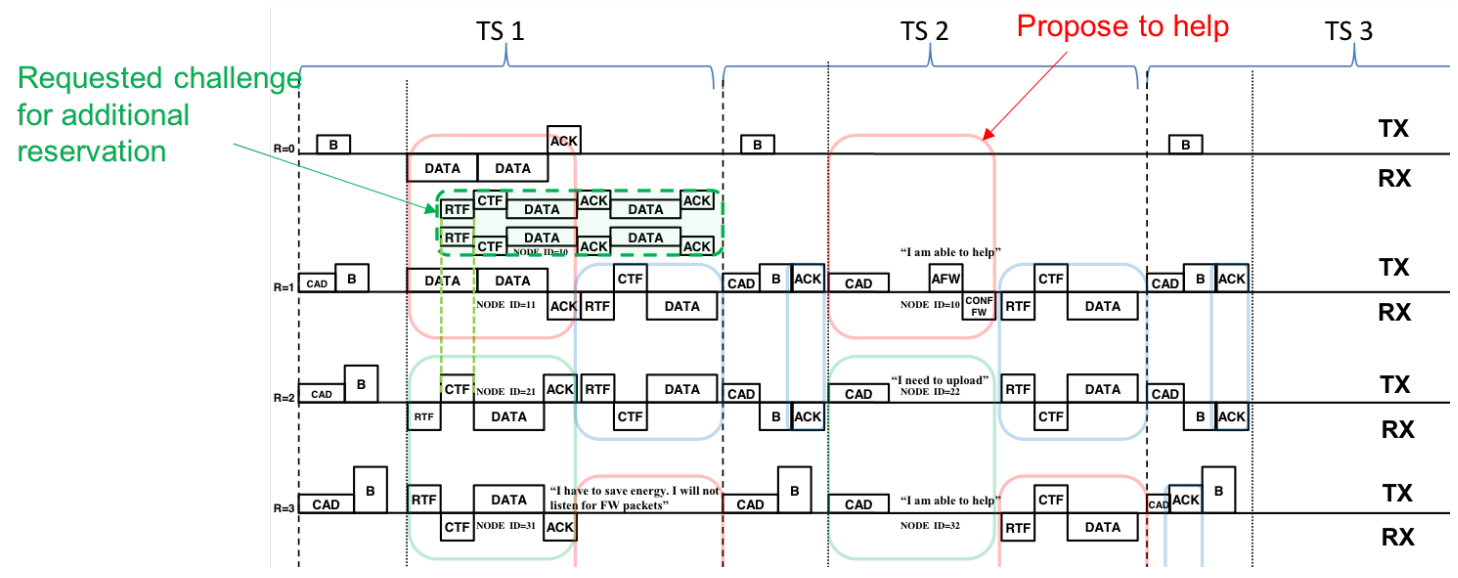

Fig. 4. Additional transmissions illustration

is sent during the Control part of the next timeslot (TS2). As we can notice, Node 11 sent 2 data packets during the 1st $\mathrm{TX} / \mathrm{RX}$ part, since it reserved its timeslot, as it was described in the previous section. Also, during Timeslot TS1, Node 10 (Rank 1) offloaded the data from its overloaded buffer through one-time on-demand slot reservation. As it was mentioned in the beginning of this chapter, the proposed MAC protocol is able to take the current battery level (SOC) of the device into account. Since Node 10 offloaded all data from its buffer and has enough energy to forward new packets from higher ranked nodes, during the next timeslot, it listens to the channel $(\mathrm{CAD})$ in order to determine whether this timeslot is reserved by another Rank 1 node. After confirming that the timeslot is free, Node 10 announces its availability to forward new packets by sending an AFW (available to forward) message to the BTS, which confirms its candidature by a forward confirmation message (CONF FW) to define one Rank 1 node per timeslot in case of multiple nodes available to forward messages. Node 22 did not receive any request to forward message, however it had its own data to send. This node used, thus, the opportunity to forward its data via Node 10. In opposition to Node 10, which had enough energy to forward additional packets, Node 31 faces a low battery issue. It cannot, thus, receive messages from the others and after transmitting its own data, it sleeps. Since the nodes take advantage of the energy harvesting module, after some time, the battery will be charged and Node 31 will be available to forward other packets.

\section{Performance eValuation}

\section{A. Simulation environment}

We implemented the WildMAC protocol in the WSNet ${ }^{4}$ event-based simulator. The performances of the WildMAC protocol are evaluated with parameter setting that coincide with our application.

\footnotetext{
${ }^{4} \mathrm{http}: / /$ wsnet.gforge.inria.fr/
}

\section{B. System sizing and parameter definition}

In this section, we assume a communication range $R$ of $R=13 \mathrm{~km}$ as obtained in previous range tests [4]. The average density of rhinoceros in the Kruger Park is $\lambda=1,7$ animals per $\mathrm{km}^{2}$. We thus distribute nodes in our simulation area using a Point Point Process with density $\lambda=1,7$ around a central point set as the base station.

In order to transmit data requested by biologists, the data packet payload size is set to 12 bytes. Control message size is set to 3 bytes.

These values along with the communication parameters chosen from LoRa (frequency $434 \mathrm{MHz}$, Spreading factor of 9 , bandwidth $31,25 \mathrm{kH}$, coding rate $4 / 8$ ) lead to the following timing configuration:

- On-air duration of a 12 byte data packet: $495.62 \mathrm{~ms}$;

- On-air duration of a control message with 3-byte payload: $233.47 \mathrm{~ms}$,

- LoRa preamble duration: $102.4 \mathrm{~ms}$.

The duration of TS was set to 3 seconds, which allows:

- 2 packet transmissions up (to the BTS) and 1 packet reception from higher ranked nodes per TS for Rank 1 devices;

- 2 transmissions up (to the BTS) for Rank 1 nodes in the one-time reserved extra slots;

The whole timeslot reservation process detailed in Section IV-D has been fully evaluated in [5] where it has been modeled as a Markov chain process. Figure 5 extracted from [5] shows the probability for a node to win the timeslot reservation process at its first attempt. From this probability, we can derive the expected number of attempts it has to perform before being successful and so the expected waiting time before accessing the channel as a function of the density.

From this study, we note that if we need $97 \%$ reservation success at first attempt, we need to have a set of minimum 80 available resources (computed as the number of available channels multiplied by the number of available timeslots in a superframe). We decide to use 4 different channels and to set $n_{T S}=20$. This leads to a superframe duration of $60 s$. 


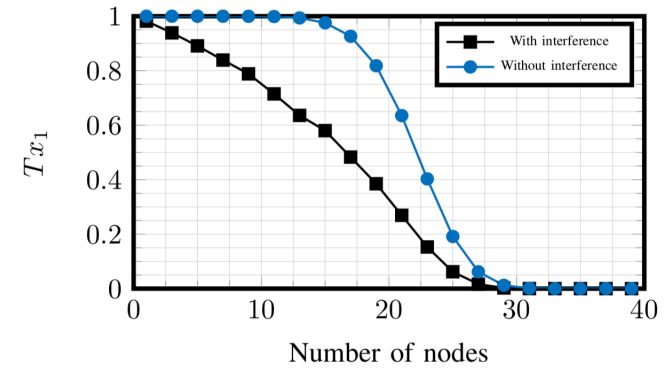

(a) Probability of transmission at the first reservation attempt

Fig. 5. Probability of transmission at the first reservation attempt. From [5]

\section{Delivery rate}

We first evaluate the packet delivery rate of regular packets, e.g. the ratio of packets successful received at the base station, as a function of their rank as shown of Fig. V-Da. We observe that the rate is very good since whatever the rank, the packet delivery rate is superior to $98 \%$, in spite of a slight decrease with the number of rank. This is due to the fact that, once a slot is reserved, thanks to the spreading factor used and the capture effect, the packet is almost sure to be received successfully. Losses that we observe are due to queue saturation. Note that this phenomenon increases with the number of ranks (cumulation of loss at each hop), which can be explained by the fact that, with only one base station, the number of nodes at rank $k$ increases with $k$. We expect this phenomenon be compensated by the presence of multiple base stations since the further a node to a base station, the higher probability to get closer to another one.

\section{Delay}

We then evaluate the end-to-end delay of regular packets. Note that the delay is averaged only for packets successfully received at the base station.

As seen in Section V-B, we defined the number of resources such that the probability to reserve a slot at first attempt is equal to $\mathrm{P}=97 \%$. If all attempts are independent, the mean delay to be observed is as follows:

$$
\text { delay }_{\text {reservation }}=\sum_{i=1}^{n_{T S}}(i-1) d \times(1-P)^{i-2} \times P
$$

where $d$ is the time between two attempts. In the best case, $d$ is equal to $d_{T S}$ and in the worst case it is equal to $d_{S F}$, depending on the node can attempt a new reservation in the same super frame or has to wait for the next one. Fig.V-Dc that plots the delay reservation and the bounds shows that globally, WildMAC behaves well between both bounds.

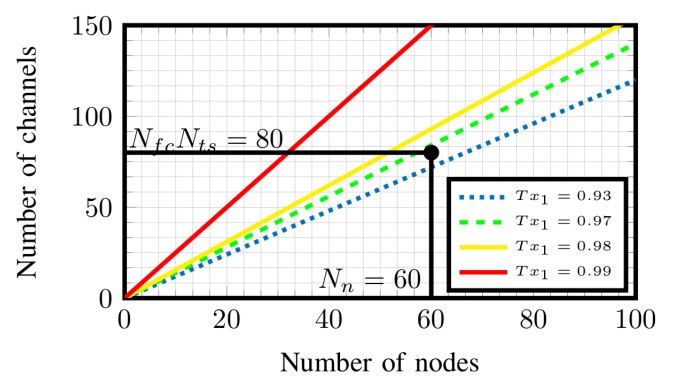

(b) Capacity
On Fig. V-Db, we can distinguish the end-to-end delay for both Alert and Regular messages. For regular messages, this delay includes the slot reservation delay (also re-plot on the figure for comparison purpose), the time spent in node's queue and the time for retransmissions when required. This delay obviously increases with the number of rank. However, we can note that the delay for rank $i$ is much less $i$ times the delay for rank 1. This is due to the fact that at each hop, the relay node does not always have to wait for the next superframe for forwarding data. It can also send aggregated data in the same superframe depending on the time slot it reserves.

Alert messages are sent in the control part of each time slot and thus can travel a full hop/range at each time slot. The delay for alert messages is thus directly equal to the number of ranks times $d_{T S}$ as expected.

In every case, we can note that all messages are received within delays required by the application (See Section II).

\section{CONCLUSION}

In this work, we have proposed a new multichannel, multihop MAC protocol for wildlife monitoring. This protocol is specific to Rhinoceros monitoring usecase but could be extended to several long range applications featuring both alert and regular messages modes. The simulation results have shown good performance in terms of delay and packet delivery rates that meet the application QoS requirements. As future work, we extend to better stress our system through experimentation and to improve energy consumption to integrate it with the other components of the rhino protection project.

\section{ACKNOWLEDGMENT}

This work has been partially supporter by a grant from CPER/FEDER DATA and the LIRIMA PredNet project. In addition, the authors would like to thank Dr Woluther and his team for the collaboration around these works. 


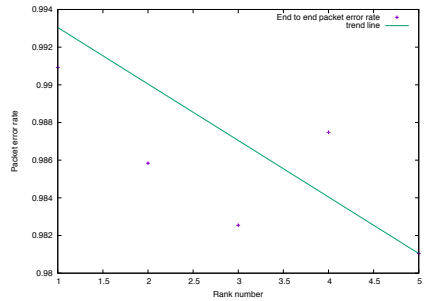

(a) Regular packet delivery rate

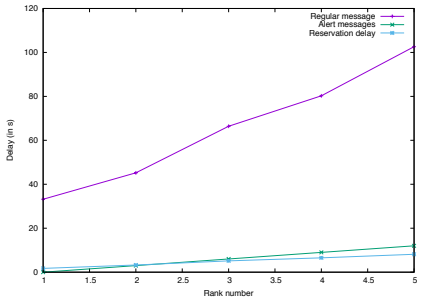

(b) End-to-end delay

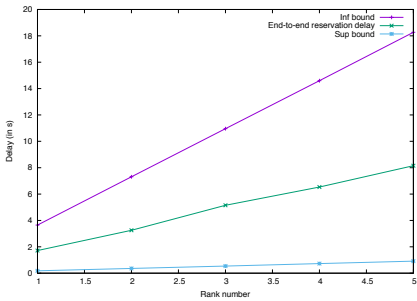

(c) End-to-end reservation delay

Fig. 6. WildMAC evaluations

\section{REFERENCES}

[1] K. Mikhaylov, J. Petaejaejaervi, and T. Haenninen, "Analysis of capacity and scalability of the lora low power wide area network technology," in European Wireless Conference, Oulu, Finland, May 2016.

[2] H. Mroue, N. Abbass, B. Parrein, S. Hamrioui, E. Motta-Cruz, and G. Rouyer, "Analytical and Experimental study for LoRa Modulation," in International Conference on Telecommunications (ICT), St. Malo, France, 2018.

[3] J. Zuniga and B. Ponsard, "SIGFOX system description," november 2016. [Online]. Available: https://www.ietf.org/id/ draft-zuniga-lpwan-sigfox-system-de-scription-01.txt

[4] V. Toldov, J. Meijers, R. Igual-Pérez, R. Wolhuter, N. Mitton, and L. Clavier, "Performance evaluation of LoRa radio solution for PREDNET wildlife animal tracking project," in LPWAN, Roissy, France, 2016.

[5] M. El Amine Seddik, V. Toldov, L. Clavier, and N. Mitton, "From Outage Probability to ALOHA MAC Layer Performance Analysis in Distributed WSNs," in Wireless Comm. and Networking Conf. (WCNC), Barcelona, Spain, 2018.

[6] F. Ferrari, M. Zimmerling, L. Thiele, and S. O., "Efficient network flooding and time synchronization with glossy," in Conference on Information Processing in Sensor Networks (IPSN), Chicago, USA, 2011.

[7] P. Sommer and Y.-A. Pignolet, "Competition: Energy-efficient network flooding with channel-hopping," in Conf. on Embedded Wireless Systems and Networks (EWSN), Uppsala, Sweden, 2017.

[8] M. Bor, J. Vidler, and U. Roedig, "Lora for the internet of things," in Conf. on Embedded Wireless Systems and Networks (EWSN), Graz, Austria, 2016.

[9] S. Fundation, "Sigfox foundation partners with three international conservation organisations to give a voice to endangered rhinos with the internet of things (iot)," March 2017. [Online]. Available: https://www.sigfox.com/en/news/ sigfox-foundation-partners-three-international-conservation-organisation

[10] S. Press, "Semtech LoRa technology tracks location of endangered black rhinos in africa," January 2017. [Online]. Available: https://www.semtech.com/company/press/

Semtech-LoRa-Technology-Tracks-Location-of-Endangered-Black-Rh
[11] IBM, "The internet of things, cloud and analytics: The new frontier in protecting the endangered rhino," September 2017. [Online]. Available: https://www.ibm.com/blogs/internet-of-things/ protecting-endangered-rhinos/

[12] P. Juang, H. Oki, Y. Wang, M. Martonosi, L. S. Peh, and D. Rubenstein, "Energy-efficient computing for wildlife tracking: Design tradeoffs and early experiences with zebranet," SIGARCH Comput. Archit. News, vol. 30 , no. 5 , pp. $96-107$, Oct. 2002.

[13] F. Dressler, S. Ripperger, M. Hierold, T. Nowak, C. Eibel, B. Cassens, F. Mayer, K. Meyer-Wegener, and A. Kolpin, "From radio telemetry to ultra-low-power sensor networks: tracking bats in the wild," IEEE Communications Magazine, vol. 54, no. 1, pp. 129-135, January 2016.
[14] G. del Campo, S. C. Sierra, J. M. Perandones, J. Song, R. Martínez, and A. Santamaria, "Poster: Improving manufacturing processes using open-iot batnet technology," in Conf. on Embedded Wireless Systems and Networks, EWSN, Uppsala, Sweden, 2017.

[15] P. Sommer, B. Kusy, P. Valencia, R. Dungavell, and R. Jurdak, "Delaytolerant networking for long-term animal tracking," IEEE Internet Computing, vol. 22, no. 1, pp. 62-72, Jan 2018.

[16] S. Toledo, O. Kishon, Y. Orchan, A. Shohat, and R. Nathan, "Lessons and experiences from the design, implementation, and deployment of a wildlife tracking system," in Conference on Software Science, Technology and Engineering (SWSTE), Beer Sheva, Israel, June 2016.

[17] D. Anthony, W. P. Bennett, M. C. Vuran, M. B. Dwyer, S. Elbaum, A. Lacy, M. Engels, and W. Wehtje, "Sensing through the continent: Towards monitoring migratory birds using cellular sensor networks," in Conf. on Information Processing in Sensor Networks (IPSN), Beijing, China, 2012.

[18] L. M. Elbroch and H. Quigley, "Social interactions in a solitary carnivore," Current Zoology, vol. 63, no. 4, pp. 357-362, 2017.

[19] M. Coyne and B. Godley, "Satellite tracking and analysis tool (stat): an integrated system for archiving, analyzing and mapping animal tracking data," Marine Ecology Progress Series, vol. 301, pp. 1-7, 2005.

[20] L. Alliance, "Lorawan specification, 2016, 70 p." 2016. [Online]. Available: https://lora-alliance.org/lorawan-for-developers

[21] M. Buettner, G. V. Yee, E. Anderson, and R. Han, "X-MAC: A short preamble MAC protocol for duty-cycled wireless sensor networks," in Conf. on Embedded Networked Sensor Systems (Sensys), Boulder, Colorado, USA, 2006.

[22] A. Dunkels, "The contikimac radio duty cycling protocol," SICS Technical Report, Tech. Rep., 2011.

[23] S. Duquennoy, O. Landsiedel, and T. Voigt, "Let the tree bloom: Scalable opportunistic routing with ORPL," in Conf. on Embedded Networked Sensor Systems (Sensys), Roma, Italy, 2013. [24] N. Accettura, L. A. Grieco, G. Boggia, and P. Camarda, "Performance
give-voianalysis of the rpl routing protocol," in IEEE International Conference on Mechatronics, 2011.

[25] P. Bahl, R. Chandra, and J. Dunagan, "Ssch: Slotted seeded channel hopping for capacity improvement in ieee 802.11 ad-hoc wireless imetworks," in International Conference on Mobile Computing and Networking (MobiCom), Philadelphia, PA, USA, 2004.

[26] K. Xie, S. He, X. Wang, D. Zhang, and K. Li, "Cooperative routing in multi-radio multi-hop wireless network," in Ad Hoc Networks, 2017.

[27] S. Duquennoy, B. Al Nahas, O. Landsiedel, and T. Watteyne, "Orchestra: Robust mesh networks through autonomously scheduled TSCH," in Conf. on Embedded Networked Sensor Systems (Sensys), Seoul, South Korea, 2015.

[28] Y. Nam, H. Lee, H. Jung, T. Kwon, and Y. Choi, "An adaptive mac (A-MAC) protocol guaranteeing network lifetime for wireless sensor networks," in EWC - Enabling Technologies for Wireless Multimedia Communications, Berlin, Germany, April 2006. 